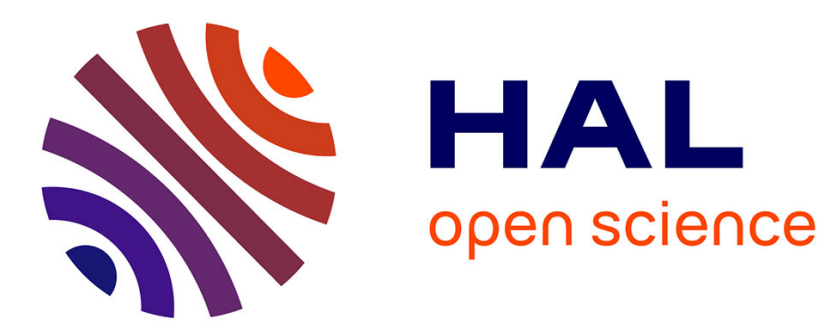

\title{
A SIMPLIFIED APPROACH TO THE REFINED BLOWUP BEHAVIOR FOR THE NONLINEAR HEAT EQUATION
}

Philippe Souplet

\section{To cite this version:}

Philippe Souplet. A SIMPLIFIED APPROACH TO THE REFINED BLOWUP BEHAVIOR FOR THE NONLINEAR HEAT EQUATION. 2018. hal-01721261

\section{HAL Id: hal-01721261 \\ https://hal.science/hal-01721261}

Preprint submitted on 1 Mar 2018

HAL is a multi-disciplinary open access archive for the deposit and dissemination of scientific research documents, whether they are published or not. The documents may come from teaching and research institutions in France or abroad, or from public or private research centers.
L'archive ouverte pluridisciplinaire HAL, est destinée au dépôt et à la diffusion de documents scientifiques de niveau recherche, publiés ou non, émanant des établissements d'enseignement et de recherche français ou étrangers, des laboratoires publics ou privés. 


\title{
A SIMPLIFIED APPROACH TO THE REFINED BLOWUP BEHAVIOR FOR THE NONLINEAR HEAT EQUATION
}

\author{
Philippe SOUPLET \\ Université Paris 13, Sorbonne Paris Cité, CNRS UMR 7539 \\ Laboratoire Analyse Géométrie et Applications \\ 93430 Villetaneuse, France. Email: souplet@math.univ-paris13.fr
}

\begin{abstract}
We consider radial decreasing solutions of the semilinear heat equation, both for subcritical and supercritical powers. We provide a much simpler and more pedagogical proof of the classical results on the sharp final blowup profile and on the refined space-time behavior in the subcritical case. We also improve some of the known results, by providing estimates in a more global form. In particular, we obtain the rate of approach of the solution to its singular final profile, given by
\end{abstract}

$$
u(x, t) \sim\left[(p-1)(T-t)+\frac{(p-1)^{2}}{8 p} \frac{|x|^{2}}{|\log | x||}\right]^{-1 /(p-1)}, \quad \text { for all } t \in\left[T-|x|^{2}, T\right)
$$

for fixed $x$ small.

\section{Introduction and main results}

We consider the classical semilinear heat equation with power nonlinearity.

$$
\left.\begin{array}{rlrl}
u_{t}-\Delta u & =|u|^{p-1} u, & & x \in \Omega, t>0, \\
u & =0, & & x \in \partial \Omega, t>0, \\
u(x, 0) & =u_{0}(x), & & x \in \Omega,
\end{array}\right\}
$$

where $\Omega \subset \mathbb{R}^{n}$ and $p>1$.

The asymptotic behavior of blowup solutions for problem (1.1) has been studied in great detail in the past thirty years (see, e.g., [34], [35], [7], [10], [8], [11], [12], [5], [13], [31], [14], [33], [2], [15], [23], [24], [19], [36], [29], [30], [20]) and the situation is rather well understood.

In particular the sharp final blowup profiles and the corresponding refined space-time behaviors have been completely classified in the Sobolev subcritical range $1<p<p_{S}:=(n+2) /(n-2)_{+}$(see [5], [13], [14], [31], [32], [24]). However, the proofs of these results are extremely long and technical, even in one space dimension or for radial decreasing solutions.

In this article we concentrate on the special but fundamental case of radial decreasing solutions, with single-point blowup, and our goal is twofold:

- Provide a much simpler and more pedagogical proof of the sharp final blowup profile and of the refined space-time behavior;

and at the same time:

- Improve some of the known results, by providing estimates in a more global form, including the rate of approach of the solution to its singular final profile. 


\subsection{The subcritical or critical case}

Our main result is the following global, refined blowup estimate, valid in the scale of the original variables $(x, t)$. In all the article we denote by $\beta=1 /(p-1)$ the scaling exponent of problem (1.1) and set $\kappa=\beta^{\beta}$.

Theorem 1.1. Let $1<p \leq p_{S}, \Omega=\mathbb{R}^{n}$, and let $u_{0} \in L^{\infty}\left(\mathbb{R}^{n}\right), u_{0} \geq 0$, be radially symmetric, nonincreasing in $|x|$, and nonconstant. Let $u$ be the unique maximal classical solution of (1.1) and assume $T:=T_{\max }\left(u_{0}\right)<\infty$. Then we have

$$
u(x, t)=(\kappa+o(1))\left[T-t+\frac{p-1}{4 p} \frac{|x|^{2}}{\min \{|\log (T-t)|, 2|\log | x||\}}\right]^{-\beta}, \quad \text { as }(x, t) \rightarrow(0, T) .
$$

Recall that, under the assumptions of Theorem 1.1 , since 0 is the only blowup point, the spaceprofile $u(x, T)=\lim _{t \rightarrow T} u(x, t)$ exists for all $x \neq 0$, as a consequence of standard parabolic estimates. As special cases, Theorem 1.1 contains the following two well-known results concerning the final blowup profile and the space-time behavior in the logarithmic scale (see [13], [14], [31] for $p<p_{S}$, and also [24]; for $p=p_{S}$, this is a consequence of [31] along with results in [10] and [19]).

Corollary A. Under the assumptions of Theorem 1.1, we have

$$
\lim _{x \rightarrow 0}\left(\frac{|x|^{2}}{|\log | x||}\right)^{\beta} u(x, T)=C_{p}:=\left[\frac{8 p}{(p-1)^{2}}\right]^{\frac{1}{p-1}}
$$

and

$$
u(\xi \sqrt{(T-t)|\log (T-t)|}, t)=(\kappa+o(1))(T-t)^{-\beta}\left[1+\frac{(p-1)|\xi|^{2}}{4 p}\right]^{-\beta},
$$

as $t \rightarrow T$, uniformly for $\xi$ bounded.

Theorem 1.1 seems new under this form. It guarantees in particular that

$$
u(x, t) \sim\left[(p-1)(T-t)+\frac{(p-1)^{2}}{8 p} \frac{|x|^{2}}{|\log | x||}\right]^{-\beta}
$$

for $t \geq T-|x|^{2}$, with fixed $x$ small, which provides information on how the solution approaches its final, singular space profile. More precisely, for each $\varepsilon \in(0,1)$, there exists $\rho_{\varepsilon}>0$ such that, for each $x$ with $|x| \leq \rho_{\varepsilon}$, we have

$$
1-\varepsilon \leq\left[(p-1)(T-t)+\frac{(p-1)^{2}}{8 p} \frac{|x|^{2}}{|\log | x||}\right]^{\beta} u(x, t) \leq 1+\varepsilon, \quad \text { for all } t \in\left[T-|x|^{2}, T\right) .
$$

In comparison with previously known proofs of Corollary A, our proof of Theorem 1.1 is considerably simpler and shorter. In particular, the upper part of estimate (1.2) requires only two pages. As for the proof of the lower part, it is about seven-page long, including the proof of the refined behavior in the self-similar range (cf. Theorem B below), which is a key intermediate step of independent interest. We refer to Section 2 for a more detailed description of the main ideas of proofs.

As mentioned above, the complete classification of final blowup profiles and refined space-time behaviors for $p<p_{S}$ (without symmetry assumptions) was obtained in [13], [14], [31], [32]. This major achievement necessitated very long and technical calculations. We note that, even though Corollary A only deals with the radial nondecreasing case, its original proof (see [14, Theorem 1] and also [1]) required this complete classification. ${ }^{1}$

On the contrary, our proofs are direct, but they pretty much depend on the radial decreasing nature of the solutions under consideration (so that we of course do not claim to recover the full strength of the original approach regarding general solutions).

\footnotetext{
${ }^{1}$ In the original proof, the irrelevant behaviors corresponding to the higher nonmonotone profiles are eventually ruled out by looking at the number of local maxima of the eigenfunctions involved in the intermediate, self-similar range asymptotics.
} 


\subsection{The supercritical case}

Since the situation is more complicated in the supercritical case, we first need to recall some known facts. Blowup is said to be type I if

$$
\limsup _{t \rightarrow T}(T-t)^{\beta}\|u(t)\|_{\infty}<\infty
$$

and type II otherwise. As for the lower bound, it is known that, in any case, $(T-t)^{\beta}\|u(t)\|_{\infty} \geq \kappa$. For nonnegative radial solutions, both type I and type II blowups occur if $n \geq 11$ and $p>p_{J L}:=$ $1+4 \frac{n-4+2 \sqrt{n-1}}{(n-2)_{+}(n-10)_{+}}$(cf. [15], [25], [21]), whereas only type I blowup occurs if $1<p<p_{J L}$ and $\Omega=B_{R}$ (cf. [11] for $p<p_{S}$, and [19], [4] for $p \geq p_{S}$ ). The latter remains true with $\Omega=\mathbb{R}^{n}$, assuming an additional assumption on $u_{0}$ if $p>p_{S}$, for instance $u_{0} \in H^{1}\left(\mathbb{R}^{n}\right)$ [20] or $u_{0}$ nonincreasing in $r$ [28].

Let us now consider the supercritical case $p>p_{S}$, in $\Omega=\mathbb{R}^{n}$ or $B_{R}$, for nonnegative radial solutions $u$ blowing-up at $x=0$. It is known from [22] and [20] that such $u$ admits a local self-similar profile $w_{\infty}$ near 0 , defined by

$$
w_{\infty}(y):=\lim _{t \rightarrow T}(T-t)^{\beta} u(y \sqrt{T-t}, t), \quad y \in \mathbb{R}^{n} \backslash\{0\} .
$$

If, moreover, blowup is type I, then the limit exists also at $y=0$ and it is uniform on compact sets. Furthermore, the following classification of $w_{\infty}$ was obtained in [20]:

- If blowup is type II, then $w_{\infty}(y) \equiv c_{p}|y|^{-2 \beta}$ (singular steady-state) and the final blowup profile in the original variable has the same asymptotics, namely $u(x, T) \sim c_{p}|x|^{-2 \beta}$ as $x \rightarrow 0$. Moreover, there exists a sequence $t=t_{j} \rightarrow T$ such that

$$
u\left(x, t_{j}\right)=(1+o(1)) m\left(t_{j}\right) U_{1}\left(x m^{\frac{p-1}{2}}\left(t_{j}\right)\right), \quad j \rightarrow \infty, \quad \text { uniformly for } x \in \Omega,
$$

where $U_{1}$ is the unique, radial regular steady state of $(1.1)$ in $\mathbb{R}^{n}$ with $U_{1}(0)=1$, and $u$ is extended by 0 outside $\Omega$ in case $\Omega=B_{R}$ (see [19], and also [4]).

- If blowup is type I, then either $w_{\infty} \equiv \kappa$ (like in the subcritical case - cf. [10], [12]) or $w_{\infty}=\varphi$, where $\varphi(y)$ is a bounded, nonconstant, radial solution of the elliptic equation $\Delta \varphi-\frac{y}{2} \cdot \nabla \varphi+\varphi^{p}-\beta \varphi=0$ in $\mathbb{R}^{n}$. Such $\varphi$ exist if $p_{S}<p<p_{L}$, where $p_{L}:=1+\frac{6}{(n-10)_{+}}>p_{J L}$ (see [16], [17]), and do not exist for $n \geq 11$ and $p \geq p_{L}$, so that this second case cannot occur (see [26], [27]). As for the final blowup profiles and refined blowup behavior, it is known [31] that $w_{\infty} \equiv \kappa$ guarantees (1.3), as well as (1.4), whereas $w_{\infty} \equiv \varphi$ implies $u(x, T) \sim L|x|^{-2 \beta}$ with $0<L \neq c_{p}$ (see [20]).

In addition (see [26]), any radial solution (type I or II) such that $w_{\infty} \not \equiv \kappa$ satisfies the upper estimate

$$
u(x, t) \leq C\left(1+|x|^{-2 \beta}\right), \quad \text { as }(x, t) \rightarrow(0, T) .
$$

We shall prove:

Theorem 1.2. Let $n \geq 3, p>p_{S}, \Omega=\mathbb{R}^{n}$ and let $u_{0} \in L^{\infty}\left(\mathbb{R}^{n}\right), u_{0} \geq 0$, be radially symmetric, nonincreasing in $|x|$, and nonconstant. Let $u$ be the unique maximal classical solution of (1.1). Assume that $T:=T_{\max }\left(u_{0}\right)<\infty$ and that

$$
w_{\infty}(y):=\lim _{t \rightarrow T}(T-t)^{\beta} u(y \sqrt{T-t}, t)=\kappa, \quad \text { for all } y \in \mathbb{R}^{n} .
$$

Then the asymptotic behavior (1.2) is true, hence in particular (1.3) and (1.4).

Remarks 1.3. (a) For $p>p_{S}$, it was already known (cf. [31]) that estimates (1.3) and (1.4) are true under the assumptions of Theorem 1.2. We here extend this to the global estimate (1.2).

(b) As a consequence of the above mentioned results, for $n \geq 11$ and $p>p_{L}$, assumption (1.7) is satisfied whenever blow-up is type I, which is generically true (see [21]).

As a curiosity, we finally mention the following proposition, which gives a natural extension, valid for all $p>1$, of the space-time upper estimate in the logarithmic scale. It has the advantage of being true regardless of the blow-up behavior of $u$. As a drawback, it need not be always sharp (see Remark 1.5). 
Proposition 1.4. Let $p>1$ and $\Omega=\mathbb{R}^{n}$ or $B_{R}, u_{0} \in L^{\infty}(\Omega), u_{0} \geq 0$, be radially symmetric and nonincreasing in $|x|$. Let $u$ be the unique maximal classical solution of $(1.1)$, assume $T:=T_{\max }\left(u_{0}\right)<\infty$ and denote $m(t):=u(0, t)=\|u(t)\|_{\infty}$. Assume in addition that $u_{0}$ is nonconstant if $\Omega=\mathbb{R}^{n}$. Then we have

$$
u(x, t) \leq(1+o(1)) m(t)\left[1+\frac{(p-1)^{2}}{4 p} \frac{|x|^{2} m^{p-1}(t)}{\min \left\{\log \left(m^{p-1}(t)\right),\left.|\log | x\right|^{2} \mid\right\}}\right]^{-\beta}, \quad \text { as }(x, t) \rightarrow(0, T)
$$

Remark 1.5. In case of type I blowup with profile $w_{\infty} \not \equiv \kappa$, property (1.5) is more precise than (1.8). Indeed, since $u_{r} \leq 0$ and $\varphi(y) \sim c|y|^{-2 \beta}$ as $|y| \rightarrow \infty$, it implies $u(x, t) \leq \varepsilon(T-t)^{-\beta}$ whenever $|x| / \sqrt{T-t} \geq K$ with $K=K(\varepsilon)>0$ large enough. However, in case of type II blowup, with $p \geq p_{J L}$, (1.8) holds at any time $t \in(0, T)$, whereas (1.6) is only known to hold for some sequence $t_{j} \rightarrow T$. On the other hand, (1.6) is more precise than (1.8) at those times $t_{j}$ (since $U(y) \sim c|y|^{-2 \beta}$ as $|y| \rightarrow \infty$ ).

\section{Ideas of proofs}

\subsection{Upper bounds}

Unlike [13], [31], [24], we will adopt two rather different approaches for proving the upper and the lower estimates. The upper estimates turn out to be easier and essentially provable by means of (sophisticated) maximum principle arguments. In particular their proof does not require Theorem B below (which is used in [13], [31], [24] for both upper and lower estimates). Namely, we apply the maximum principle to the functional

$$
J:=u_{r}+\frac{r u^{p}}{2 p(A+\log u)}
$$

where $A>0$ is a sufficiently large constant, and then use careful analysis of the integrated version of the resulting inequality $J \leq 0$. This is an improvement of a device from [9] (which was itself a refinement on a method in [7]). Actually, in [9], the functional (2.1) was used with $A=0$ instead, and applied to an auxiliary problem with positive boundary values and particular initial data. Then intersection-comparison with problem (1.1) was used to obtain the upper part of the final space profile estimate (1.3), requiring an additional intersection number assumption on $u_{0}$ and the restriction $p<p_{S}$ (see Theorem 7.3 and final remark p.815 in [9]). Here, the different choice of $J$ enables one to work directly on the original problem (1.1). In this way, no additional restriction on $u_{0}$ is needed, any $p>1$ is allowed, and we can obtain the upper part of the new space-time estimate (1.8) as well.

\subsection{Lower bounds}

As for the proofs of the lower estimates, we remain closer to the general approach in [13], [31], although our proofs are also significantly simpler. Namely, as a preliminary step to (1.4), of independent interest, the following result was obtained in [5], [14], [32] (see also [6], [1], [18]). It gives a more precise expansion of the solution than (1.4), but only in the more restricted range $|x| \leq C \sqrt{T-t}$.

Theorem B. Let $p>1, \Omega=\mathbb{R}^{n}$ and $\rho(y)=e^{-|y|^{2} / 4}$. Let $u_{0}$ be as in Theorem 1.2 and assume (1.7). Then we have

$$
(T-t)^{\beta} u(y \sqrt{T-t}, t)=\kappa\left(1-\frac{|y|^{2}-2 n+o(1)}{4 p|\log (T-t)|}\right), \quad \text { as } t \rightarrow T,
$$

with convergence in $H_{\rho}^{1}\left(\mathbb{R}^{n}\right)$ and uniformly for $y$ bounded. 
The original proofs of Theorem B are quite long and technical, even for the one dimensional or radial situations. We shall here provide a proof of Theorem B which is significantly simpler than the previously known ones, although it uses some of their fundamental ideas, relying on self-similar change of variables and dynamical system arguments for the transformed equation. It is well known from [10], [8], [11], [12] that the function $w(y, s):=e^{-\beta s} u\left(e^{-s / 2} y, T-e^{-s}\right)=(T-t)^{\beta} u(y \sqrt{T-t}, t)$ is a global solution of the rescaled equation

$$
w_{s}-\Delta w+\frac{1}{2} y \cdot \nabla w=w^{p}-\beta w
$$

and that, if $p<p_{S}$, then $w(y, s)$ is bounded and converges to $\kappa$ as $s \rightarrow \infty$, uniformly for $y$ bounded. To determine the next term in the asymptotic expansion (cf. (2.2)), the idea (cf. [5], [14]) is to linearize equation (2.3) around $w=\kappa$ and perform a kind of center manifold analysis with respect to the linear operator $-\Delta+\frac{1}{2} y \cdot \nabla$ acting on $L_{\rho}^{2}$. In a radial framework, this leads to the Ansatz $w-\kappa:=$ $a(s)+b(s)\left(|y|^{2}-2 n\right)+\theta(y, s)$, where the decomposition is orthogonal in $L_{\rho}^{2}$, and where $b(s)\left(|y|^{2}-2\right)$ and $\theta$ are respectively the neutral and stable components.

A key step in the proof of Theorem B is to show non-exponential decay ${ }^{2}$ of $w(\cdot, s)$ to $\kappa$ in $L_{\rho}^{2}$. Actually, as a main novelty, we obtain in Lemma 5.3 the lower bound $\|w(\cdot, s)-\kappa\|_{L_{\rho}^{2}} \geq c s^{-1}$ as a simple consequence of the maximum principle argument leading to the upper part of (1.2). Note that in [14], even the $1 \mathrm{~d}$ radial decreasing case required the complete classification of higher nonmonotone profiles and that, in the absence of Lemma 5.3 on non-exponential decay, the corresponding behaviors could only be eventually ruled out by looking at the number of local maxima of the higher eigenfunctions. Also, once non-exponential decay is established, as further simplifications in our current framework of radial nondecreasing solutions, we can reduce the proof of Theorem B to some simple linear differential inequalities by means of suitable weighted Poincaré inequalities (cf. Proposition 5.1 and 5.2).

Finally, to deduce the lower parts of Theorems 1.1 and 1.2 from Theorem B, one has to extend the lower part of estimate (2.2) from the region $|y| \sim 1$ to the larger range of the original variables $(x, t)$. To do so, we use and simplify the idea from [14], which combines a finely tuned rescaling procedure and a comparison argument. ${ }^{3}$ Namely, the lower bound provided by Theorem B is used as a new initial condition for a rescaled equation, starting at a suitable intermediate time, whose solution is then estimated from below by a specific susbsolution. Let us mention that the rescaled solution is given by

$$
v_{\sigma}(z, \tau)=\sigma^{\beta} u(z \sqrt{\sigma}, T-\sigma+\sigma \tau), \quad z \in \mathbb{R}^{n}, 0 \leq \tau<1
$$

and that we find an optimal choice of the parameters and variables, given by $\sigma=\max \left\{2(T-t),|x|^{2}\right\}$, $z=x \sigma^{-1 / 2}$ and $\tau=1-(T-t) \sigma^{-1}$ (for fixed $(x, t)$ close to $(0, T)$ ), which yields the lower part of the new global estimate (1.2).

The outline of the rest of the paper is as follows. In Section 2, we prove Proposition 1.4 and deduce the upper part of estimate (1.2) in Theorems 1.1-1.2. In Section 3, we prove the lower part of estimate (1.2) in Theorems 1.1-1.2, assuming Theorem B. We note that these two sections are quite short. A relatively longer step remains the proof of Theorem B itself, which is carried out in Section 4. Finally in Appendix, for self-containedness, we prove some weighted Poincaré and Poincaré-Wirtinger type inequalities that are used in the proof of Theorem B.

\footnotetext{
${ }^{2}$ Note that exponential decay would heuristically mean dominance of $\theta$ instead of $b(s)\left(y^{2}-2 n\right)$, and that such (unstable) behavior may occur in radial nonmonotone situations, leading to different blowup profiles.

${ }^{3}$ Let us point out that a different method for such derivations was introduced in [24], based on a Liouville-type theorem from [23], which has the advantage to avoid maximum principle arguments and hence be extendible to systems. However such Liouville-type theorems only hold for subcritical $p$.
} 


\section{Proofs of upper estimates}

We first prove Proposition 1.4, from which the upper estimates in Theorems 1.1, 1.2 and Corollary A will follow easily.

Proof of Proposition 1.4. We set $f(u):=u^{p}$ and $R=1$ in case $\Omega=\mathbb{R}^{n}$. Since $u \geq e^{-t A} u_{0}$, there exists $\eta>0$ such that

$$
u(x, t) \geq \eta>0 \quad \text { in } D:=\bar{B}_{R / 2} \times[T / 2, T) .
$$

Setting $\Omega_{1}:=\Omega \cap\left\{x: x_{1}>0\right\}$, we notice that $v:=u_{x_{1}} \leq 0$ satisfies $v_{t}-\Delta v=f^{\prime}(u) v \leq 0$ in $\Omega_{1}$. Since $v=0$ for $x \in \partial \Omega_{1} \cap\left\{x_{1}=0\right\}$, the strong maximum principle and the Hopf Lemma imply $v<0$ for $x \in \Omega_{1}$ and $u_{r r}(0, t)=v_{x_{1}}(0, t)<0$ for $t>0$. Therefore, there exists $k>0$ such that

$$
u_{r} \leq-k r \text { on } \partial_{P} Q
$$

where $Q=(0, R / 2) \times(T / 2, T)$.

We consider the auxiliary function $J:=u_{r}(r, t)+c(r) F(u)$. We compute

$$
\left[\partial_{t}-\partial_{r}^{2}\right](c F(u))=c F^{\prime}(u)\left(u_{t}-u_{r r}\right)-c F^{\prime \prime}(u) u_{r}^{2}-2 c^{\prime} F^{\prime}(u) u_{r}-c^{\prime \prime} F(u)
$$

in $Q$, and

$$
\left[\partial_{t}-\partial_{r}^{2}\right] u_{r}=\frac{n-1}{r} u_{r r}-\frac{n-1}{r^{2}} u_{r}+f^{\prime}(u) u_{r}
$$

Omitting the variables $r, t, u$ when no confusion arises, it follows that

$$
J_{t}-J_{r r}=\frac{n-1}{r} u_{r r}-\frac{n-1}{r^{2}} u_{r}+f^{\prime} u_{r}+c F^{\prime}\left(\frac{n-1}{r} u_{r}+f\right)-c F^{\prime \prime} u_{r}^{2}-2 c^{\prime} F^{\prime} u_{r}-c^{\prime \prime} F .
$$

Substituting $u_{r}=J-c F$ and $u_{r r}=J_{r}-c^{\prime} F-c F^{\prime} u_{r}=J_{r}-c F^{\prime} J+c^{2} F F^{\prime}-c^{\prime} F$, we obtain

$$
\begin{aligned}
J_{t}-J_{r r}= & \frac{n-1}{r}\left(J_{r}-c F^{\prime} J+c^{2} F F^{\prime}-c^{\prime} F\right)-\frac{n-1}{r^{2}}(J-c F)+f^{\prime}(J-c F) \\
& +c F^{\prime}\left(\frac{n-1}{r}(J-c F)+f\right)-c F^{\prime \prime}(J-c F)^{2}-2 c^{\prime} F^{\prime}(J-c F)-c^{\prime \prime} F .
\end{aligned}
$$

Setting

$$
\mathcal{P} J:=J_{t}-J_{r r}-\frac{n-1}{r} J_{r}+b J, \quad \text { with } b:=\frac{n-1}{r^{2}}-f^{\prime}-c F^{\prime \prime}(2 c F-J)+2 c^{\prime} F^{\prime},
$$

it follows that

$$
\begin{aligned}
\mathcal{P} J & =\frac{n-1}{r}\left(c^{2} F F^{\prime}-c^{\prime} F\right)+\frac{n-1}{r^{2}} c F-c F f^{\prime}+c F^{\prime}\left(-\frac{n-1}{r} c F+f\right)-c^{3} F^{\prime \prime} F^{2}+2 c c^{\prime} F F^{\prime}-c^{\prime \prime} F \\
& =c\left(F^{\prime} f-F f^{\prime}\right)+\frac{n-1}{r^{2}}\left(c-r c^{\prime}\right) F-c^{3} F^{\prime \prime} F^{2}+2 c c^{\prime} F F^{\prime}-c^{\prime \prime} F .
\end{aligned}
$$

Now choose $c(r)=r$ and $F(u)=f(u) \phi(u)$, where $f(u):=u^{p}$ and the function $\phi \in C^{2}([\eta, \infty))$, to be determined, satisfies

$$
\phi>0, \quad \phi^{\prime} \leq 0, \quad(f \phi)^{\prime \prime} \geq 0 \quad \text { in }[\eta, \infty) .
$$

We have in $Q$ :

$$
\mathcal{P} J \leq F^{\prime} f-F f^{\prime}+2 F^{\prime} F=f\left(f^{\prime} \phi+f \phi^{\prime}\right)-f f^{\prime} \phi+2 f \phi\left(f^{\prime} \phi+f \phi^{\prime}\right) \leq f^{2} \phi^{\prime}+2 f f^{\prime} \phi^{2} .
$$

A sufficient condition for $\mathcal{P} J \leq 0$ is thus

$$
-\frac{\phi^{\prime}}{\phi^{2}}=\frac{2 f^{\prime}}{f}=\frac{2 p}{u} \Longleftrightarrow \frac{1}{\phi}=2 p(A+\log u) \quad(A=\text { Const }) .
$$


Choosing any positive constant $A>-\log \eta$, this yields $\phi(u)=\frac{1}{2 p(A+\log u)}$ i.e., $J=u_{r}+\frac{r u^{p}}{2 p(A+\log u)}$. A straightforward computation shows that (3.3) is true, provided we further assume that $A \geq-\log \eta+$ $\frac{2 p-1}{p(p-1)}$. By $(3.1)$, we have

$$
r^{-1} J \leq-k+\frac{u^{p}}{2 p(A+\log \eta)} \quad \text { in }(0, R / 2] \times[T / 2, T) .
$$

Since we already know that 0 is the unique blowup point (see [7] and [3], for the cases $\Omega=B_{R}$ and $\Omega=\mathbb{R}^{n}$ respectively), we may choose $A$ sufficiently large so that $J \leq 0$ on the parabolic boundary of $Q$. Since the coefficient $b$ in (3.2) is bounded from below for $t$ bounded away from $T$, we may apply the maximum principle to deduce that $J \leq 0$ i.e.,

$$
-u^{-p}(A+\log u) u_{r} \geq \frac{r}{2 p}
$$

By integration, we are left with $\int_{u(r, t)}^{u(0, t)} s^{-p}(A+\log s) d s \geq \frac{r^{2}}{4 p}$ in $Q$. Integrating by parts, we arrive at

$$
\left[s^{1-p}\left(A+\frac{1}{p-1}+\log s\right)\right]_{u(0, t)}^{u(r, t)} \geq \frac{(p-1) r^{2}}{4 p} .
$$

Setting $m(t):=u(0, t) \rightarrow \infty$ as $t \rightarrow T$ and $G(s)=s^{1-p}\left(A+\frac{1}{p-1}+\log s\right)$, it follows that

$$
G[u(r, t)] \geq G[m(t)]+\frac{(p-1) r^{2}}{4 p} \geq \frac{(p-1) r^{2}}{4 p} .
$$

The function $G(s)$ decreases monotonically to 0 as $s \rightarrow \infty$, and it is easy to show that its inverse function $G^{-1}$ is well-defined and decreasing on some interval $\left(0, \delta_{0}\right]$, and satisfies

$$
G^{-1}(X) \sim \kappa X^{-\frac{1}{p-1}}|\log X|^{\frac{1}{p-1}}, \quad X \rightarrow 0^{+}
$$

By (3.5), we have

$$
u(r, t) \leq G^{-1}\left(G(m(t))+\frac{(p-1) r^{2}}{4 p}\right) \leq G^{-1}\left(\frac{(p-1) r^{2}}{4 p}\right) \quad \text { in } \tilde{D}:=\left(0, r_{0}\right] \times[T / 2, T)
$$

for some $r_{0}>0$, In particular, taking $r_{0}$ smaller if necessary, we have

$$
u(r, t) \leq G^{-1}\left(m^{1-p}(t) \log (m(t))+\frac{(p-1) r^{2}}{4 p}\right) \quad \text { in }\left[0, r_{0}\right] \times\left[T-r_{0}, T\right) .
$$

Also, we may assume $m^{1-p}(t) \leq m^{1-p}(t) \log (m(t))+\frac{p-1}{4 p} r^{2}<1$, hence

$$
\left|\log \left(m^{1-p}(t) \log m(t)+\frac{(p-1) r^{2}}{4 p}\right)\right| \leq \min \left((p-1) \log (m(t)),\left|\log \left(\frac{(p-1) r^{2}}{4 p}\right)\right|\right) .
$$

For each $\rho>0$ small, it follows that

$$
\begin{aligned}
u(r, t) & \leq(1+\varepsilon(\rho)) \kappa\left[\frac{m^{1-p}(t) \log m(t)+\frac{(p-1) r^{2}}{4 p}}{\left|\log \left(m^{1-p}(t) \log m(t)+\frac{(p-1) r^{2}}{4 p}\right)\right|}\right]^{-\beta} \\
& \leq(1+\varepsilon(\rho)) \kappa\left[\frac{m^{1-p}(t)}{p-1}+\frac{p-1}{4 p} \frac{r^{2}}{\min \{(p-1) \log (m(t)), 2|\log r|\}}\right]^{-\beta}
\end{aligned}
$$

in $[0, \rho] \times[T-\rho, T)$, with $\lim _{\rho \rightarrow 0} \varepsilon(\rho)=0$. Estimate (1.8) follows. 
Proofs of upper estimates in Theorems 1.1-1.2 and Corollary A. Since $m(t)=u(0, t)$, we have $m^{\prime}(t) \leq m^{p}$ by $(1.1)$, hence $m(t) \geq \kappa(T-t)^{-\beta}$ by integration. If $p<p_{S}$, this along with [10] guarantees

$$
m(t)=(1+o(1)) \kappa(T-t)^{-\beta}, \quad t \rightarrow T .
$$

If $p=p_{S}$, we know that blowup is type I by [19], so that (3.8) still follows from the main theorem in [10]. If $p>p_{S}$ (Theorem 1.2), we have (3.8) by assumption. In all cases, the upper part part of estimate (1.2) is then a direct consequence of (1.8). The upper estimates in (1.3) and (1.4) immediately follow.

\section{Proofs of lower estimates assuming Theorem B}

In this section, we deduce the lower parts of Theorems 1.1 and 1.2 from Theorem B. The goal is thus to extend the lower estimate, given by Theorem B in the region $|y| \sim 1$ to the range of the original variables $(x, t)$. To do so, we use and simplify the idea from [14], which combines a finely tuned rescaling procedure and a comparison argument. Namely, the lower bound provided by Theorem B is used as a new initial condition for a rescaled equation, starting at a suitable intermediate time, and the solution of the rescaled equation is then estimated from below by a specific susbsolution. We note that this argument does not directly use the radial symmetry of $u$.

Proof of lower part of estimate (1.2) in Theorems 1.1 and 1.2. By Theorem B, we may write

$$
(T-t)^{\beta} u(x, t)=\kappa\left(1-\frac{x^{2}-2 n(T-t)}{4 p(T-t)|\log (T-t)|}\right)+\frac{\mathcal{R}\left(x(T-t)^{-1 / 2},|\log (T-t)|\right)}{|\log (T-t)|},
$$

with $\lim _{s \rightarrow \infty}\|\mathcal{R}(\cdot, s)\|_{H_{\rho}^{1}}=0$. We introduce a rescaling parameter $\sigma \in(0,1)$ and define

$$
v_{\sigma}(z, \tau)=\sigma^{\beta} u(z \sqrt{\sigma}, T-\sigma+\sigma \tau), \quad z \in \mathbb{R}^{n}, 0 \leq \tau<1 .
$$

Suitable values of $\sigma$ will be selected later. The function $v_{\sigma}$ solves $\partial_{\tau} v_{\sigma}-\Delta_{z} v_{\sigma}=v_{\sigma}^{p}$ in $\mathbb{R}^{n} \times(0,1)$. Considering its initial data, we see that

$$
v_{\sigma, 0}(z):=v_{\sigma}(z, 0)=\sigma^{\beta} u(z \sqrt{\sigma}, T-\sigma)=\kappa\left(1-\frac{|z|^{2}-2 n}{4 p|\log \sigma|}\right)+\frac{\mathcal{R}(z,|\log \sigma|)}{|\log \sigma|} .
$$

Denote by $G_{\tau}(x)=(4 \pi \tau)^{-n / 2} \exp \left(-|x|^{2} / 4 \tau\right)$ the Gaussian heat kernel and set $e^{-\tau A} v_{\sigma, 0}=G_{\tau} * v_{\sigma, 0}$. For each fixed $\sigma$, we consider the comparison function

$$
V_{\sigma}(z, \tau)=\left[\left(e^{-\tau A} v_{\sigma, 0}\right)^{1-p}(z)-(p-1) \tau\right]^{-\beta} .
$$

The function $V_{\sigma}$ is well defined in $\mathbb{R}^{n} \times\left(0, \tau_{0}\right)$ for some small $\tau_{0}>0$, due to $v_{\sigma, 0} \in L^{\infty}\left(\mathbb{R}^{n}\right)$. By direct computation, we check that $\partial_{\tau} V_{\sigma}-\Delta_{z} V_{\sigma} \leq V_{\sigma}^{p}$, as long as $V_{\sigma}$ exists, with $V_{\sigma}(\cdot, 0)=v_{\sigma, 0}$. It follows from the comparison principle that $V_{\sigma}$ exists in $\mathbb{R}^{n} \times(0,1)$ and that

$$
v_{\sigma}(z, \tau) \geq V_{\sigma}(z, \tau) \text { in } \mathbb{R}^{n} \times(0,1) .
$$

By a simple calculation involving Gaussians and integration by parts ${ }^{4}$ we find that $G_{\tau} *\left(|z|^{2}-2 n\right)=$ $|z|^{2}-2 n(1-\tau)$, hence

$$
\left(e^{-\tau A} v_{\sigma, 0}\right)(z)=\kappa\left(1-\frac{|z|^{2}-2 n(1-\tau)}{4 p|\log \sigma|}\right)+\frac{\left[e^{-\tau A} \mathcal{R}(\cdot,|\log \sigma|)\right](z)}{|\log \sigma|} .
$$

\footnotetext{
${ }^{4}$ It may seem easier to use the fact that $|z|^{2}-2 n(1-\tau)$ solves the heat equation, but this requires to invoke a uniqueness result in classes of functions which grow at infinity.
} 
To control the second term on the RHS, we observe that, for all $\phi \in L_{\rho}^{2}$,

$$
\left|\left(e^{-\tau A} \phi\right)(z)\right| \leq C(n)\|\phi\|_{L_{\rho}^{2}} \tau^{-n / 2} e^{|z|^{2} / 2}, \quad z \in \mathbb{R}^{n}, 0<\tau \leq 1
$$

Indeed, using the identity $\frac{1}{4}|y-z|^{2}+\frac{1}{2}|z|^{2}-\frac{1}{6}|y|^{2}=\frac{1}{12}|y-3 z|^{2} \geq 0$ and the Cauchy-Schwarz inequality, we obtain,

$$
(4 \pi \tau)^{n / 2}\left|\left(e^{-\tau A} \phi\right)(z)\right| \leq \int|\phi(y)| e^{-\frac{|y-z|^{2}}{4 \tau}} d y \leq e^{\frac{|z|^{2}}{2}} \int|\phi(y)| e^{-\frac{|y|^{2}}{6}} d y \leq C e^{\frac{|z|^{2}}{2}}\left(\int|\phi(y)|^{2} e^{-\frac{y^{2}}{4}} d y\right)^{1 / 2} .
$$

Fix $\varepsilon>0$. Since $\lim _{s \rightarrow \infty}\|\mathcal{R}(\cdot, s)\|_{L_{\rho}^{2}}=0$, we deduce from the above that there exists $\sigma_{0}=\sigma_{0}(\varepsilon) \in$ $(0,1 / 2]$ such that, for all $\sigma \in\left(0, \sigma_{0}\right]$ and $\tau \in[1 / 2,1)$,

$$
\left(e^{-\tau A} v_{\sigma, 0}\right)(z) \geq \kappa\left(1-\frac{|z|^{2}-2 n(1-\tau)+\varepsilon}{4 p|\log \sigma|}\right) \geq \kappa\left(1-\frac{|z|^{2}+\varepsilon}{4 p|\log \sigma|}\right)>0, \quad|z| \leq 1 .
$$

Taking $\sigma_{0}(\varepsilon)$ smaller if necessary, if follows from (4.2) that, for all $|z| \leq 1$ and $\tau \in[1 / 2,1)$,

$$
\begin{aligned}
v_{\sigma}(z, \tau) & \geq\left[\kappa^{1-p}\left(1-\frac{|z|^{2}+\varepsilon}{4 p|\log \sigma|}\right)^{1-p}-(p-1) \tau\right]^{-\beta} \\
& =\kappa\left[\left(1-\frac{|z|^{2}+\varepsilon}{4 p|\log \sigma|}\right)^{1-p}-\tau\right]^{-\beta} \geq \kappa\left[1-\tau+\frac{p-1}{4 p} \frac{|z|^{2}+2 \varepsilon}{|\log \sigma|}\right]^{-\beta}
\end{aligned}
$$

where we used $\kappa=(p-1)^{-1 /(p-1)}$ and $(1-h)^{1-p} \leq 1+(p-1) h+C(p) h^{2}$ for $h \in[0,1 / 2]$. Going back to $u$ through (4.1), we have thus obtained that, for all $\sigma \in\left(0, \sigma_{0}\right]$ and $\tau \in[1 / 2,1)$,

$$
u(z \sqrt{\sigma}, T-\sigma+\sigma \tau) \geq \kappa\left[\sigma(1-\tau)+\frac{p-1}{4 p} \frac{\sigma\left(|z|^{2}+2 \varepsilon\right)}{|\log \sigma|}\right]^{-\beta}, \quad|z| \leq 1 .
$$

Now, for each $(x, t) \in B_{\sqrt{\sigma}_{0}} \times\left[T-\sigma_{0} / 2, T\right)$, we select

$$
\sigma=\max \left\{2(T-t),|x|^{2}\right\} \in\left(0, \sigma_{0}\right], \quad z=x \sigma^{-1 / 2}, \quad \tau=1-(T-t) \sigma^{-1} \in\left[\frac{1}{2}, 1\right),
$$

hence $|z| \leq 1$ and $T-\sigma+\sigma \tau=t$. Consequently, we obtain

$$
u(x, t) \geq \kappa\left[T-t+\frac{p-1}{4 p} \frac{|x|^{2}}{|\log \sigma|}+\frac{\varepsilon \sigma}{|\log \sigma|}\right]^{-\beta} .
$$

Since $\sigma \leq 2(T-t)+|x|^{2}$, it follows that

$$
u(x, t) \geq \frac{\kappa}{(1+C(p) \varepsilon)^{\beta}}\left[T-t+\frac{p-1}{4 p} \frac{|x|^{2}}{\min \{|\log (2(T-t))|, 2|\log | x||\}}\right]^{-\beta},
$$

whenever $0<|x|<\sqrt{\sigma_{0}(\varepsilon)}$ and $t \in\left[T-\sigma_{0}(\varepsilon) / 2, T\right)$.

Proof of lower estimates in Corollary A. Since 0 is the only blow-up point, it follows from interior parabolic estimates that $u(x, T)$ is well defined for all $x \neq 0$. The lower part of $(1.3)$ is then as easy consequence of the lower part of (1.2).

Next, fixing $K, \varepsilon>0$ and considering $|x|^{2} \leq K(T-t)|\log (T-t)|$, we have $\left|\log \left(|x|^{2}\right)\right| \geq(1-$ $\varepsilon)|\log (T-t)|$ as $t \rightarrow T$, hence the lower part of (1.2) readily implies the lower estimate in (1.4). 


\section{Proof of Theorem B}

\subsection{Preliminaries}

We consider $w$, the rescaled solution in similarity variables around $(0, T)$ :

$$
w(y, s):=e^{-\beta s} u\left(e^{-s / 2} y, T-e^{-s}\right), \quad y:=\frac{x}{\sqrt{T-t}}, \quad s:=-\log (T-t),
$$

where $\left.w(y, s)=(T-t)^{\beta} u(x, t)\right)$. Let $s_{0}:=-\log T$. Then $w$ is a global solution of

$$
w_{s}-\Delta w+\frac{1}{2} y \cdot \nabla w=|w|^{p-1} w-\beta w . \quad y \in \mathbb{R}^{n}, s \in\left(s_{0}, \infty\right),
$$

Under the assumptions of Theorem B, we have in particular $w \leq C$ and it is known from [10], [8], [11], [12] that

$$
\lim _{s \rightarrow \infty} w(y, s)=\kappa, \quad \text { uniformly for } y \text { bounded. }
$$

Recast in terms of $w$, the desired estimate (2.2) is equivalent to

$$
w(y, s)=\kappa\left(1-\frac{|y|^{2}-2 n}{4 p s}\right)+o\left(\frac{1}{s}\right)
$$

as $s \rightarrow \infty$, with convergence in $H_{\rho}^{1}\left(\mathbb{R}^{n}\right)$ and uniformly for $y$ bounded. Here we are using the spaces

$$
L_{\rho}^{2}:=\left\{v \in L_{l o c}^{2}\left(\mathbb{R}^{n}\right): \int_{\mathbb{R}^{n}} v^{2}(y) \rho(y) d y<\infty\right\}, \quad H_{\rho}^{1}:=\left\{f \in L_{\rho}^{2}: \nabla f \in L_{\rho}^{2}\right\}
$$

where $\rho(y):=e^{-|y|^{2} / 4}$. We respectively denote by $(v, w):=\int_{\mathbb{R}^{n}} u v \rho d y$ and $\|v\|=(v, v)^{1 / 2}$ the inner product and the norm of the Hilbert space $L_{\rho}^{2}$ and we set $\|v\|_{H_{\rho}^{1}}^{2}=\left(\|v\|^{2}+\|\nabla v\|^{2}\right)^{1 / 2}$.

The idea to show (5.4) is to linearize equation (5.2) around $w=\kappa$ and perform a kind of center manifold analysis. However, as noted in [5], standard center manifold theory cannot be directly applied, since $L_{\rho}^{2}$, which is the natural space for the problem, does not have the required properties. Although the basic strategy is similar, our proof (in the radial decreasing case) is somewhat simpler and shorter than the original proofs.

As a pedagogical preparation to the rigorous proof, we first recall the sketch of the formal proof of Theorem B for $p=2$ and $n=1$ (cf. [5, pp. 828-829]). In this case, the equation (5.2) for $w$ is just $w_{s}=w_{y y}-\frac{1}{2} y w_{y}+w^{2}-w$ and $\varphi:=\kappa-w=1-w$ thus solves the simple equation

$$
\varphi_{s}=\varphi_{y y}-\frac{1}{2} y \varphi_{y}+\varphi-\varphi^{2}, \quad y \in \mathbb{R}, s>0 .
$$

On the other hand, $\varphi$ is not just any solution: we know from the above that $\varphi$ is globally bounded and decays to 0 locally uniformly as $s \rightarrow \infty$. Next, it can be checked (see Remark 6.2) that the linearized operator $\mathcal{L}_{0}=\partial_{y}^{2}-\frac{1}{2} y \partial_{y}+1$, acting on $L_{\rho}^{2}$ (with natural domain) and restricted to symmetric functions, has:

- one unstable direction, corresponding to constant eigenfunctions;

- one neutral direction, colinear to the quadratic eigenfunction $y^{2}-2$; and

- a stable subspace of codimension two.

It is thus natural to make the Ansatz $\varphi=a(s)+b(s)\left(y^{2}-2\right)+\theta(y, s)$, where the decomposition is orthogonal in $L_{\rho}^{2}$, and where one expects that the coefficients $a, b$ decay to 0 as $s \rightarrow \infty$. The goal is then to show that the neutral mode $b(s)\left(y^{2}-2\right)$ dominates. Intuitively, $a(s)$ cannot be dominant, 
since otherwise the leading equation would be $a^{\prime} \sim a$, leading to unboundedness of $\varphi$. On the contrary, dominance of the stable component $\theta$ is expected to lead to an exponential decay of $\varphi$. However, it can be shown that this does not occur in the symmetric decreasing case; see Lemma 5.3. We are thus left with the scenario $\varphi \sim b(s)\left(y^{2}-2\right)$ as $s \rightarrow \infty$. Testing the equation with $\left(y^{2}-2\right) \rho$, we see that the linear terms on RHS disappear, due to $y^{2}-2$ belonging to the kernel of the operator $\mathcal{L}_{0}$, and this leads to

$$
b^{\prime}(s) \sim-c_{2} b^{2}, \quad \text { where } c_{2}=\left(\int\left(y^{2}-2\right) \rho d y\right)^{-1} \int\left(y^{2}-2\right)^{3} \rho d y .
$$

The precise behavior $b(s) \sim c_{2} s^{-1}$ follows by integration and this completes the formal proof.

In the rigorous proof, the dominance of the neutral mode will be obtained by establishing differential inequalities satisfied by the weighted $L^{2}$ norm of $\varphi$, of its gradient, and of its respective projections on the unstable, neutral and stable subspaces. The various linear and nonlinear terms will be suitably handled in the $L_{\rho}^{2}$ functional framework, by means of the following simple weighted Poincaré and Poincaré-Wirtinger type inequalities:

Proposition 5.1. We have

$$
\int_{\mathbb{R}^{n}}|y|^{2} v^{2} \rho d y \leq 16\|\nabla v\|^{2}+4 n\|v\|^{2}, \quad \text { for all } v \in H_{\rho}^{1}
$$

Proposition 5.2. Let $v \in H_{\rho}^{1}$.

(i) If $\left(v, y_{j}\right)=0$ for all $j \in\{1, \ldots, n\}$, then we have

$$
\|v\|^{2} \leq\|\nabla v\|^{2}+\bar{v}^{2}, \quad \text { where } \bar{v}=\left(\int_{\mathbb{R}^{n}} \rho d y\right)^{-1 / 2} \int_{\mathbb{R}^{n}} v \rho d y
$$

In particular, (5.6) is true whenever $v$ is radially symmetric.

(ii) If $v$ is orthogonal to all polynomials of degree $\leq 3$, then we have

$$
\|v\|^{2} \leq \frac{1}{2}\|\nabla v\|^{2}
$$

In particular, (5.7) is true whenever $v$ is radially symmetric and $(v, 1)=\left(v,|y|^{2}\right)=0$.

(iii) Let $i \in\{1, \ldots, n\}$ and assume $\partial_{y_{i}} v \in H_{\rho}^{1}$. If $\left(v, y_{i}\right)=\left(v, y_{i}^{2}-2\right)=0$ and $\left(v, y_{i} y_{j}\right)=0$ for all $j \neq i$, then

$$
\left\|\partial_{y_{i}} v\right\|^{2} \leq\left\|\nabla\left(\partial_{y_{i}} v\right)\right\|^{2}
$$

In particular, (5.8) is true for all $i \in\{1, \ldots, n\}$ whenever $v$ is radially symmetric, $\nabla v \in H_{\rho}^{1}$ and $\left(v,|y|^{2}-2 n\right)=0$.

Propositions 5.1 and 5.2 are direct consequences of well-known spectral properties of the Hermite operator $\mathcal{L} v=-\Delta v+\frac{y}{2} \cdot \nabla v \mathrm{n} H_{\rho}^{1}$. Altough this material may be considered as standard, complete proofs of these properties are given in Appendix for convenience and self-containedness. We point out that, although the higher eigenfunctions of $\mathcal{L}$ are completely known (given by Hermite polynomials, see Proposition 6.1 and Remark 6.2), our proof does not require to consider any decomposition of $\theta$. 


\subsection{A simple direct proof of nonexponential decay of $w-\kappa$}

The following lemma provides a polynomial lower bound on the decay of $\kappa-w$ in $L_{\rho}^{2}$. As explained in the above heuristic argument, this will turn out to be an important piece of information. This lower bound is obtained as a simple consequence of the maximum principle argument leading to the upper part of (1.2), that we already proved.

Lemma 5.3. Under the assumptions of Theorem 1.1, there exists $c>0$ such that

$$
\|\kappa-w(\cdot, s)\|_{L_{\rho}^{2}} \geq c s^{-1}, \quad s \rightarrow \infty
$$

Proof. By (3.4), we know that $u=u(r, t), r=|x|$, satisfies, for some constants $A, c_{1}>0$,

$$
-u_{r} \geq c_{1} r \frac{u^{p}}{A+\log u}, \quad 0 \leq r \leq 1 / 2, T / 2<t<T .
$$

Fix $R>0$. By (5.3), there exists $t_{0}=t_{0}(R) \in(T / 2, T)$ such that

$$
u(r, t) \geq \frac{\kappa}{2}(T-t)^{-\beta}, \quad 0 \leq r \leq R \sqrt{T-t}, t_{0}<t<T
$$

Consider $\varphi=\kappa-w$, with $w=w(\rho, s)$ and $\rho=|y|$. Observing that the RHS of (5.9) is an increasing function of $u$ for large $u$, we deduce that, for $s_{0}=s_{0}(R)$ sufficiently large,

$$
\begin{aligned}
\varphi_{\rho}(\rho, s) & =-w_{\rho}(\rho, s)=-(T-t)^{\beta+\frac{1}{2}} u_{r}(\rho \sqrt{T-t}, t) \\
& \geq \frac{c_{1} \rho(T-t)^{\beta+1}\left(\frac{\kappa}{2}\right)^{p}(T-t)^{-p \beta}}{A+\log \left(\frac{\kappa}{2}(T-t)^{-\beta}\right)}=\frac{c_{1}\left(\frac{\kappa}{2}\right)^{p} \rho}{A+\log \frac{\kappa}{2}+\beta s} \geq \frac{c_{2} \rho}{s}
\end{aligned}
$$

for all $0 \leq \rho \leq R$ and $s>s_{0}$, where the constant $c_{2}>0$ is independent of $R$.

Now, choose $R=2\left(1+c_{2}^{-1}\right)$ and take any $s>s_{0}(R)$. If $\varphi(1, s) \geq-1 / s$, then it follows that

$$
\varphi(\rho, s)=\varphi(1, s)+\int_{1}^{\rho} \varphi_{\rho}(z, s) d z \geq \frac{-1+c_{2}(\rho-1)}{s} \geq \frac{1}{s}, \quad R-1 \leq \rho \leq R,
$$

hence $\|\varphi(s)\|_{L_{\rho}^{2}} \geq\left(\int_{R-1}^{R} \rho\right)^{1 / 2} s^{-1}$. Otherwise, we have $\varphi(1, s) \leq-1 / s$ and, since $\varphi$ is a nondecreasing function of $\rho$, we get $\varphi(\rho, s) \leq-1 / s$ for $\rho \in[0,1]$, hence $\|\varphi(s)\|_{L_{\rho}^{2}} \geq\left(\int_{0}^{1} \rho\right)^{1 / 2} s^{-1}$. We conclude that $\|\varphi(s)\|_{L_{\rho}^{2}} \geq c s^{-1}$ for all $s \geq s_{0}$.

\subsection{Proof of Theorem B}

Recall $(v, w)=\int_{\mathbb{R}^{n}} v w \rho d y$ and set $\|v\|=(v, v)^{1 / 2}$. Integrals over $\mathbb{R}^{n}$ will be simply denoted by $\int$ and the variables will be omitted when no confusion arises. For clarity we split the proof into several steps.

Step 1. Preliminaries and decomposition of $\kappa-w$. Set $\varphi=\kappa-w$. Note that, under the current assumptions, $\varphi$ is radially symmetric nondecreasing, and that $\varphi<\kappa$. By (5.2), using $\kappa=\beta^{\beta}$, we see that $\varphi$ solves the equation

$$
\varphi_{s}+\mathcal{L} \varphi=\varphi-F(\varphi), \quad \text { where } F(\varphi)=(\kappa-\varphi)^{p}-\kappa^{p}+p \kappa^{p-1} \varphi
$$

and $\mathcal{L} \varphi=-\Delta \varphi+\frac{y}{2} \cdot \nabla \varphi=-\rho^{-1} \nabla \cdot(\rho \nabla \varphi)$. 
Also we have $-C \leq \varphi<\kappa$, as a consequence of (1.7). Moreover, by parabolic estimates, one can obtain

$$
\sup _{\mathbb{R}^{n} \times\left(s_{1}, s_{2}\right)}\left(|D w|+\left|D^{2} w\right|+\left|D^{3} w\right|+(1+|y|)^{-1}\left(\left|w_{s}\right|+\left|\nabla w_{s}\right|\right)\right)<\infty, \quad s_{0}<s_{1}<s_{2},
$$

which provide polynomial bounds on the derivatives of $\varphi$. In view of the exponential decay of $\rho$, these bounds will guarantee the validity of the integrations by parts and differentiations under the integral sign that we will carry out in the rest of the proof.

Moreover, for each $R>0$,

$$
m_{R}(s):=\sup _{|y| \leq R}|\varphi(y, s)| \rightarrow 0, \quad \text { as } s \rightarrow \infty
$$

and $\lim _{s \rightarrow \infty}\|\varphi(\cdot, s)\|=0$, as a consequence of (1.7) and dominated convergence. Next we observe that

$$
0 \leq F(\varphi) \leq C \varphi^{2}
$$

Indeed, by Taylor's formula with integral remainder, for $X<\kappa, X \neq 0$, we have

$$
V(X):=\frac{F(X)}{X^{2}}=p(p-1) \int_{0}^{1}(\kappa-t X)^{p-2}(1-t) d t .
$$

If $p \geq 2$, since $\varphi$ is bounded, this immediately implies (5.13). If $1<p<2$, we have $0<\int_{0}^{1}(\kappa-$ $t \varphi)^{p-2}(1-t) d t \leq \int_{0}^{1} \kappa^{p-2}(1-t)^{p-1} d t=C$, due to $\varphi<\kappa$, hence again (5.13).

Now set $H_{0}=c_{0}$ and $H_{2}=c_{2} P$ with $P(y)=|y|^{2}-2 n$. We may choose the normalization constants $c_{0}, c_{2}$ so that $\left\|H_{0}\right\|=\left\|H_{2}\right\|=1$ and $H_{0} \perp H_{2}$. We then define the orthogonal decomposition of $\varphi$ into "unstable", "neutral" and "stable" components as follows:

$$
\varphi=a(s) H_{0}+b(s) H_{2}(y)+\theta(y, s),
$$

where $a(s):=\left(\varphi(\cdot, s), H_{0}\right), b(s):=\left(\varphi(\cdot, s), H_{2}\right)$ and $\theta:=\varphi-a(s) H_{0}-b(s) H_{2}(y)$. Consequently, we have $\theta(\cdot, s) \perp H_{0}, \theta(\cdot, s) \perp H_{2}$. Substituting the decomposition (5.15) in the PDE (5.10) and using $\mathcal{L} H_{2}=H_{2}$, we get

$$
a^{\prime}(s) H_{0}+b^{\prime}(s) H_{2}(y)+\theta_{s}+\mathcal{L} \theta=a(s) H_{0}+\theta-F(\varphi) .
$$

Integrating by parts, we obtain $\left(\mathcal{L} \theta, H_{i}\right)=\left(\theta, \mathcal{L} H_{i}\right)=(i / 2)\left(\theta, H_{i}\right)=0$ for $i=0,2$. Taking scalar products and using the orthogonality relations, it follows that

$$
a^{\prime}(s)=a(s)-c_{0} \int F(\varphi) \rho, \quad b^{\prime}(s)=-\int F(\varphi) H_{2} \rho .
$$

In the sequel, we will denote by $\varepsilon(s)$ various functions such that $\lim _{s \rightarrow \infty} \varepsilon(s)=0$.

Step 2. Control of the unstable mode in $L_{\rho}^{2}$. We shall show that

$$
|a(s)|=o(\|\varphi(s)\|), \quad s \rightarrow \infty .
$$

Set $J(s)=\int \varphi^{2} \rho$ and $K(s)=\int|\nabla \varphi|^{2} \rho$. The idea is to derive a simple differential inequality for the quantity $a^{2}-\lambda J$.

Fix any $\lambda \in(0,1 / 2)$. As a consequence of the weighted Poincaré inequality for radial functions in Proposition 5.2(i) we first have the relation

$$
J \leq a^{2}+K
$$


Testing equation (5.10) with $\rho \varphi$, we obtain $\frac{1}{2} J^{\prime}(s)=-K+J-\int F(\varphi) \varphi \rho$ hence, in view of (5.17),

$$
\frac{1}{2}\left(a^{2}-\lambda J\right)^{\prime}=a^{2}+\lambda(K-J)-c_{0} a(s) \int F(\varphi) \rho+\lambda \int F(\varphi) \varphi \rho .
$$

We proceed to show that the (nonlinear) integral terms in (5.20) are of lower order as $s \rightarrow \infty$. First note that

$$
\left|\int F(\varphi) \varphi \rho\right|+\left|a(s) \int F(\varphi) \rho\right| \leq C \int|\varphi|^{3} \rho
$$

by (5.13) and Hölder's inequality. To estimate $\int|\varphi|^{3} \rho$, we then apply the weighted Poincaré inequality in Proposition 5.1:

$$
\int \varphi^{2}|y|^{2} \rho \leq C \int\left(\varphi^{2}+|\nabla \varphi|^{2}\right) \rho,
$$

along with the boundedness of $\varphi$, to write:

$$
\int|\varphi|^{3} \rho=\int_{|y| \leq R}|\varphi|^{3} \rho+\int_{|y|>R}|\varphi|^{3} \rho \leq m_{R}(s) J+\frac{C}{R^{2}} \int_{|y|>R} \varphi^{2}|y|^{2} \rho \leq m_{R}(s) J+C R^{-2}(J+K) .
$$

For any $\eta>0$, first choosing $R=\eta^{-1 / 2}$ and then using (5.12), we obtain $\int|\varphi|^{3} \rho \leq 2 C \eta(J+K)$ for all sufficiently large $s$, hence

$$
\int|\varphi|^{3} \rho \leq \varepsilon(s)(J+K)
$$

Now, by combining (5.19)-(5.21) and (5.23), we obtain $\frac{1}{2}\left(a^{2}-\lambda J\right)^{\prime} \geq a^{2}+(\lambda-\varepsilon(s)) K-(\lambda+\varepsilon(s)) J \geq$ $(1-\lambda+\varepsilon(s)) a^{2}-2 \varepsilon(s) J \geq \frac{1}{2}\left(a^{2}-\lambda J\right)$ for $s$ large. We deduce that $a^{2}-\lambda J \leq 0$ as $s \rightarrow \infty$, since otherwise $a^{2}-\lambda J$ would grow exponentially, contradicting the boundedness of $\varphi$. Since this is true for any $\lambda \in(0,1 / 2)$, it follows that $a^{2}=o(J)$, as $s \rightarrow \infty$, which is equivalent to (5.18).

Step 3. Control of the stable component in $L_{\rho}^{2}$. We shall show that

$$
\|\theta(s)\|=o(|b(s)|), \quad s \rightarrow \infty .
$$

This time we set $L(s)=\int \theta^{2} \rho, M(s)=\int|\nabla \theta|^{2} \rho$ and the idea is to derive a simple differential inequality for the quantity $L-\lambda b^{2}$.

As a consequence of the orthogonality of the decomposition (5.15) in $L_{\rho}^{2}$, also taking into account $\left(\nabla H_{2}, \nabla \theta\right)=\left(\mathcal{L} H_{2}, \theta\right)=\left(H_{2}, \theta\right)=0$, we have

$$
J=a^{2}+b^{2}+L, \quad K=b^{2}+M .
$$

Moreover, since $\theta$ is radial and $\theta \perp H_{0}, \theta \perp H_{2}$, we may apply the better Poincaré inequality in Proposition 5.2(ii) to get

$$
L \leq \frac{1}{2} M
$$

We now test (5.16) with $\theta \rho$. Using $H_{2} \perp H_{0}, H_{0} \perp \theta(\cdot, s), H_{2} \perp \theta(\cdot, s)$ and noting that $\left(\mathcal{L} \theta, H_{2}\right)=$ $\left(\nabla H_{2}, \nabla \theta\right)=0$, we obtain $\frac{1}{2} L^{\prime}(s)=-M+L-\int F(\varphi) \theta \rho$. Fixing any $\lambda>0$, we deduce from $(5.17)$ that

$$
\frac{1}{2}\left(L-\lambda b^{2}\right)^{\prime}(s)=-M+L-\int F(\varphi) \theta \rho+\lambda b(s) \int F(\varphi) H_{2} \rho .
$$

As in Step 2, we wish to control the integral terms in (5.27). To this end, for each $\eta>0$, we write

$$
\begin{aligned}
\left|\int F(\varphi) \theta \rho\right|+\left|b(s) \int F(\varphi) H_{2} \rho\right| & \leq \eta \int \theta^{2} \rho+C_{\eta} \int \varphi^{4} \rho+C|b(s)| \int \varphi^{2}\left(|y|^{2}+1\right) \rho \\
& \leq \eta L+\left[C_{\eta} \varepsilon(s)+C|b(s)|\right](J+K),
\end{aligned}
$$


where we used (5.13), the boundedness of $\varphi,(5.23)$ and (5.22). Also, owing to (5.18), (5.19) and (5.25), we observe that $J+K+L \leq C\left(b^{2}+M\right)$. Since $\lim _{s \rightarrow \infty} b(s)=0$, we deduce that

$$
\left|\int F(\varphi) \theta \rho\right|+\left|b(s) \int F(\varphi) H_{2} \rho\right| \leq \varepsilon(s)\left(b^{2}+M\right) .
$$

Now, this along with (5.26) and (5.27) guarantees that

$$
\frac{1}{2}\left(L-\lambda b^{2}\right)^{\prime} \leq-M+L+\varepsilon(s)\left(M+b^{2}\right) \leq-(1-2 \varepsilon(s)) L+\varepsilon(s) b^{2} .
$$

Therefore, $\left(L-\lambda b^{2}\right)^{\prime} \leq-\left(L-\lambda b^{2}\right)$ for $s$ large. We deduce that $L \leq \lambda b^{2}+C e^{-s}$ as $s \rightarrow \infty$. But since, on the other hand, $J \geq c s^{-2}$ by Lemma 5.3, this along with (5.18) and (5.25), guarantees that $b^{2} \geq c s^{-2}$ as $s \rightarrow \infty$, hence (5.24).

Step 4. Control of the stable component in $H_{\rho}^{1}$. We shall show that

$$
\|\nabla \theta(s)\|=o(|b(s)|) \quad \text { as } s \rightarrow \infty .
$$

We proceed similarly as for Step 3, this time working at the level of the equation satisfied by $\partial_{y_{i}} \varphi$. We will derive a differential inequality for $M-\lambda b^{2}$.

Fix any $i \in\{1, \ldots, n\}$. Differentiating (5.10), we see that $\varphi_{i}:=\partial_{y_{i}} \varphi$ satisfies

$$
\partial_{s} \varphi_{i}+\mathcal{L} \varphi_{i}=\frac{1}{2} \varphi_{i}+G\left(\varphi, \varphi_{i}\right), \text { with } G\left(\varphi, \varphi_{i}\right):=p\left[(\kappa-\varphi)^{p-1}-\kappa^{p-1}\right] \varphi_{i} .
$$

Differentiating the decomposition in (5.15), we get $\varphi_{i}=2 c_{2} b(s) y_{i}+\theta_{i}(y, s)$, where $\theta_{i}=\partial_{y_{i}} \theta$. Substituting in the last equation and using $\mathcal{L} y_{i}=\frac{1}{2} y_{i}$, we obtain

$$
2 c_{2} b^{\prime}(s) y_{i}+\partial_{s} \theta_{i}+\mathcal{L} \theta_{i}=\frac{1}{2} \theta_{i}+G\left(\varphi, \varphi_{i}\right) .
$$

Since $\theta \perp H_{2}$, it follows from the weighted Poincaré inequality in Proposition 5.2 (iii) that

$$
M=\|\nabla \theta\|^{2} \leq N:=\sum_{i}\left\|\nabla \theta_{i}\right\|^{2} .
$$

Now fix $\lambda>0$. Testing equation (5.30) with $\theta_{i} \rho$, summing over $i$ and using (5.17), we get

$$
\frac{1}{2}\left(M-\lambda b^{2}\right)^{\prime}=-N+\frac{1}{2} M+\sum_{i} \int G\left(\varphi, \varphi_{i}\right) \theta_{i} \rho+\lambda b(s) \int F(\varphi) H_{2} \rho .
$$

To estimate the first integral term (the second was already estimated in (5.28)), we first note that, by a similar argument as for (5.13), we have $\left|G\left(\varphi, \varphi_{i}\right)\right| \leq C\left|\varphi \varphi_{i}\right|$. Then, for each $\eta>0$, we write

$$
\left|\int G\left(\varphi, \varphi_{i}\right) \theta_{i} \rho\right| \leq C \int\left|\varphi \varphi_{i} \theta_{i}\right| \rho \leq \eta \int\left(|\nabla \theta|^{2}+|\nabla \varphi|^{4}\right) \rho+C_{\eta} \int \varphi^{4} \rho .
$$

Using the boundedness of $\varphi, \nabla \varphi,(5.23), J+K+L \leq C\left(b^{2}+M\right)$ and (5.26), we deduce that $\left|\int G\left(\varphi, \varphi_{i}\right) \theta_{i}\right| \leq \varepsilon(s)\left(N+b^{2}\right)$. From (5.31), (5.32), (5.28), we then obtain $\frac{1}{2}\left(M-\lambda b^{2}\right)^{\prime} \leq-N+$ $\frac{1}{2} M+\varepsilon(s)\left(N+b^{2}\right) \leq-\frac{1}{4}\left(M-\lambda b^{2}\right)$. Therefore, $M \leq \lambda b^{2}+C e^{-s / 2}$ as $s \rightarrow \infty$. Since $b^{2} \geq c s^{-2}$ as $s \rightarrow \infty$ (cf. the end of Step 3), we deduce that $M=o\left(b^{2}\right)$ i.e., (5.29). 
Step 5. Computation of the decay rate of b and convergence in $H_{\rho}^{1}$. We shall show that

$$
\lim _{s \rightarrow \infty} s b(s)=\frac{\kappa}{4 p c_{2}} .
$$

Note that, owing to (5.25) and Steps 2 and 3, we have $|a|=o(|b|)$, hence, by Step 4,

$$
\left\|\varphi(s)-b(s) H_{2}\right\|_{H_{\rho}^{1}}=\left(a^{2}(s)+\|\theta(s)\|_{H_{\rho}^{1}}^{2}\right)^{1 / 2}=o(|b(s)|) .
$$

Property (5.33) will thus guarantee the $H_{\rho}^{1}$ convergence in the statement of the theorem, cf. (5.4).

To prove (5.33), going back to (5.17), we compute $\frac{b^{\prime}}{b^{2}}=-\int V(\varphi)\left(\frac{\varphi}{b}\right)^{2} H_{2} \rho$, where $V$ is given by (5.14). Since $\varphi(s) \sim b(s) H_{2}$, we expect that $\frac{b^{\prime}}{b^{2}} \sim-\ell$, with $\ell=V(0) \int H_{2}^{3} \rho$.

To justify this rigorously, we proceed as follows. Fix any $R>0$ and observe that $\tilde{m}_{R}(s):=$ $\sup _{|y| \leq R}|V(0)-V(\varphi(y, s))| \rightarrow 0$ as $s \rightarrow \infty$ by (5.12) and the continuity of $V$.

We write

$$
\frac{b^{\prime}}{b^{2}}(s)+\ell=\int(V(0)-V(\varphi))\left(\frac{\varphi}{b}\right)^{2} H_{2} \rho+V(0) \int\left(\left(\frac{\varphi}{b}\right)^{2}-H_{2}^{2}\right) H_{2} \rho \equiv T_{1}+T_{2} .
$$

Let us first estimate $T_{1}$. Setting $\rho_{1}=\left(1+|y|^{2}\right) \rho$, using the boundedness of $V(\varphi)(\mathrm{cf}$. (5.13)), (5.22) and the Cauchy-Schwarz inequality, we get

$$
\begin{aligned}
\left|T_{1}\right| & \leq \int_{|y| \leq R}|V(0)-V(\varphi)|\left(\frac{\varphi}{b}\right)^{2}\left|H_{2}\right| \rho \\
& \leq C \tilde{m}_{R}(s) \int_{|y| \leq R}\left(\frac{\varphi}{b}\right)^{2} \rho_{1}+C \int_{|y|>R}\left|\left(\frac{\varphi}{b}\right)^{2}-H_{2}^{2}\right| \rho_{1}+C \int_{|y|>R}\left|H_{2}\right|^{3} \rho \\
& \leq C \frac{\tilde{m}_{R}(s)}{b^{2}}\|\varphi\|_{H_{\rho}^{1}}^{2}+C\left\|\frac{\varphi}{b}-H_{2}\right\|_{H_{\rho}^{1}}\left(\left\|\frac{\varphi}{b}\right\|_{H_{\rho}^{1}}+\left\|H_{2}\right\|_{H_{\rho}^{1}}\right)+C \int_{|y|>R}\left|H_{2}\right|^{3} \rho .
\end{aligned}
$$

Next, by (5.22) and the Cauchy-Schwarz inequality, we see that $\left|T_{2}\right|$ is bounded by the second term in the last line of the last inequality. Letting $s \rightarrow \infty$, we thus obtain $\varlimsup_{s \rightarrow \infty}\left|\frac{b^{\prime}}{b^{2}}(s)+\ell\right| \leq C \int_{|y|>R}\left|H_{2}\right|^{3} \rho$, hence $\lim _{s \rightarrow \infty} \frac{b^{\prime}}{b^{2}}(s)=-\ell$ by letting $R \rightarrow \infty$. After integration, we end up with $\lim _{s \rightarrow \infty} s b(s)=\ell^{-1}$. Finally, by a straightforward calculation, using $V(0)=\frac{p(p-1)}{2} \kappa^{p-2}=\frac{p}{2 \kappa}$, we see that

$$
\ell=\frac{p c_{2}^{3}}{2 \kappa} \int P^{3} \rho=\frac{4 p c_{2}^{3}}{\kappa} \int P^{2} \rho=\frac{4 p c_{2}}{\kappa} .
$$

Step 6. Convergence in $L_{l o c}^{\infty}$. Going back to (5.16) and using (5.17), we write

$$
\theta_{s}+\mathcal{L} \theta=\theta-F(\varphi)+c_{0}^{2} \int F(\varphi) \rho+\left(\int F(\varphi) H_{2} \rho\right) H_{2}(y)
$$

Fix $R>0$. For all $|y| \leq R$, we have $|\theta|=\left|\varphi-a(s) H_{0}-b(s) H_{2}\right| \leq C(R)$. Owing to (5.33), (5.34), it follows that

$$
|F(\varphi)| \leq C\left(\theta+a H_{0}+b H_{2}\right)^{2} \leq C \theta^{2}+C\left(a H_{0}+b H_{2}\right)^{2} \leq C(R)\left(|\theta|+s^{-2}\right) .
$$

Using Proposition 5.1, we then obtain, for all $(y, s) \in Q\left(s_{0}\right):=B_{R} \times\left[s_{0}, s_{0}+1\right]$,

$$
\left|\theta_{s}+\mathcal{L} \theta\right| \leq C(R)\left(|\theta|+s_{0}^{-2}\right)+C\|\varphi(s)\|_{H_{\rho}^{1}}^{2}\left(1+|y|^{2}\right) \leq C(R)\left(|\theta|+s_{0}^{-2}\right) .
$$

Now fix $\varepsilon>0$. By Step 3, for any $s_{0}$ sufficiently large, we have $\|\theta\|_{L^{m}\left(Q\left(s_{0}\right)\right)} \leq C(R) \varepsilon s_{0}^{-1}$ with $m=2$. By interior parabolic $L^{q}$ estimates and a simple bootstrap argument on $m$, we can then show that this remains true for $m=\infty$. 


\section{Appendix. Weighted Poincaré inequalities: proof of Propositions 5.1 and 5.2}

We first give the:

Proof of Proposition 5.1. Using the identity

$$
\left(v^{2} y\right) \cdot \nabla \rho=\nabla \cdot\left(v^{2} y \rho\right)-\rho \nabla \cdot\left(v^{2} y\right)=\nabla \cdot\left(v^{2} y \rho\right)-n \rho v^{2}-2 \rho v y \cdot \nabla v
$$

and integrating on $B_{R}$, we have

$$
\begin{aligned}
\int_{B_{R}} v^{2}|y|^{2} \rho & =-2 \int_{B_{R}}\left(v^{2} y\right) \cdot \nabla \rho=2 n \int_{B_{R}} \rho v^{2}+4 \int_{B_{R}} \rho v y \cdot \nabla v+2 R \int_{\partial B_{R}} \rho v^{2} d \sigma \\
& \leq 2 n \int_{B_{R}} \rho v^{2}+\frac{1}{2} \int_{B_{R}} v^{2}|y|^{2} \rho+8 \int_{B_{R}}|\nabla v|^{2} \rho+2 R \int_{\partial B_{R}} \rho v^{2} d \sigma .
\end{aligned}
$$

Since $\rho v^{2} \in L^{1}\left(\mathbb{R}^{n}\right)$ and $\int_{\mathbb{R}^{n}} \rho v^{2} d y=\int_{0}^{\infty} \int_{\partial B_{r}} \rho v^{2} d \sigma d r$, there exists a sequence $R_{j} \rightarrow \infty$ such that $R_{j} \int_{\partial B_{R_{j}}} \rho v^{2} d \sigma \rightarrow 0$. Letting $R=R_{j} \rightarrow \infty$, we obtain (5.5).

As for Proposition 5.2, it will be conveniently proved as a consequence of properties of the following elliptic operator. For each $v \in H_{\rho}^{1}$, we define $\mathcal{L} v=-\Delta v+\frac{y}{2} \cdot \nabla v=-\rho^{-1} \nabla \cdot(\rho \nabla v)$ as the element of the dual $\left(H_{\rho}^{1}\right)^{\prime}$, given by

$$
\langle\mathcal{L} v, w\rangle:=(\nabla v, \nabla w)=\int_{\mathbb{R}^{n}} \rho \nabla v \cdot \nabla w d y, \quad \text { for all } w \in H_{\rho}^{1} .
$$

We then consider $\mathcal{L}$ as an unbounded operator on $L_{\rho}^{2}$ with domain of definition $D(\mathcal{L})=\left\{v \in H_{\rho}^{1}\right.$ : $\left.\mathcal{L} v \in L_{\rho}^{2}\right\}$. For $\lambda \in \mathbb{R}$, we say that $v \in H_{\rho}^{1}$ is an eigenfunction of $\mathcal{L}$ with eigenvalue $\lambda$ if

$$
(\nabla u, \nabla w) \equiv \int_{\mathbb{R}^{n}} \rho \nabla v \cdot \nabla w d y=\lambda(u, w) \quad \text { for all } w \in H_{\rho}^{1} .
$$

By interior elliptic regularity, any eigenfunction belongs to $C^{\infty}\left(\mathbb{R}^{n}\right)$ and satisfies

$$
-\Delta v+\frac{y}{2} \cdot \nabla v=\lambda v, \quad y \in \mathbb{R}
$$

Conversely, if $v \in C^{2}\left(\mathbb{R}^{n}\right)$ is a solution of $(6.2)$ and belongs to $H_{\rho}^{1}$, then it is not difficult to check that it is an eigenfunction. We have the following spectral results concerning the operator $\mathcal{L}$. Although it is well-known, we shall give a proof for self-containedness.

Proposition 6.1. (i) There exists a Hilbert basis of $L_{\rho}^{2}$ made of eigenfunctions of $\mathcal{L}$.

(ii) The eigenvalues of $\mathcal{L}$ are given by $\lambda_{k}=k / 2, k \in \mathbb{N}$.

(iii) For $n=1$, the eigenspaces $E_{k}=\operatorname{Ker}\left(\mathcal{L}-\lambda_{k} I\right)$ are of dimension one. They are of the form $E_{k}=\operatorname{Span}\left(P_{k}\right)$, where $P_{k}$ is a polynomial of degree $k$.

(iv) For $n \geq 1$, the eigenspace $E_{k}=\operatorname{Ker}\left(\mathcal{L}-\lambda_{k} I\right)$ is generated by the polynomials

$$
Q_{\beta}(y)=\prod_{i=1}^{n} P_{\beta_{i}}\left(y_{i}\right), \quad \beta \in \mathbb{N}^{n}, \beta_{1}+\cdots+\beta_{n}=k,
$$

where the $P_{j}$ are given in assertion (iii). In particular $E_{k}$ consists of polynomials of degree $\leq k$. 
Remark 6.2. The eigenfunctions $P_{k}$ can be expressed in terms of Hermite polynomials (see, e.g., [5]). However we shall not use this fact. We just note that for $k=0,1,2$, the eigenspace $E_{k}$ is generated by:

$$
\begin{array}{ll}
\text { if } k=0: & 1 \\
\text { if } k=1: & y_{i} \quad \text { for } 1 \leq i \leq n \\
\text { if } k=2: & y_{i}^{2}-2 \quad \text { for } 1 \leq i \leq n \quad \text { and } \quad y_{i} y_{j} \quad \text { for } 1 \leq i<j \leq n .
\end{array}
$$

For the proof of Proposition 6.1, we need the following lemma.

Lemma 6.3. The imbedding $H_{\rho}^{1} \subset L_{\rho}^{2}$ is compact.

Proof. Let $\left(v_{j}\right)$ be a bounded sequence in $H_{\rho}^{1}$. There exists a subsequence, still denoted $\left(v_{j}\right)$, and $v \in H_{\rho}^{1}$ such that $v_{j} \rightarrow v$ weakly in $H_{\rho}^{1}$. By Rellich's theorem, we may assume that $v_{j} \rightarrow v$ strongly in $L_{l o c}^{2}$. For each $R>0$, using (5.5), we write

$$
\begin{aligned}
\left\|v_{j}-v\right\|^{2} & =\int_{|y| \leq R}\left|v_{j}-v\right|^{2} \rho d y+\int_{|y|>R}\left|v_{j}-v\right|^{2} \rho d y \\
& \leq \int_{|y| \leq R}\left|v_{j}-v\right|^{2} \rho d y+R^{-2} \int_{|y|>R}|y|^{2}\left|v_{j}-v\right|^{2} \rho d y \\
& \leq \int_{|y| \leq R}\left|v_{j}-v\right|^{2} \rho d y+C R^{-2}\left(\left\|v_{j}\right\|_{H_{\rho}^{1}}^{2}+\|v\|_{H_{\rho}^{1}}^{2}\right) \leq \int_{|y| \leq R}\left|v_{j}-v\right|^{2} \rho d y+C R^{-2}
\end{aligned}
$$

Fix $\varepsilon>0$. Choosing $R=R_{0}(\varepsilon)>0$ large enough, we have $\left\|v_{j}-v\right\|^{2} \leq \int_{|y| \leq R_{0}(\varepsilon)}\left|v_{j}-v\right|^{2} \rho d y+\varepsilon$ for all $j$. Since $v_{j} \rightarrow v$ strongly in $L_{l o c}^{2}$, we then have $\left\|v_{j}-v\right\|^{2} \leq 2 \varepsilon$ for all large $j$. Therefore $v_{j} \rightarrow v$ strongly in $L_{\rho}^{2}$ and the lemma is proved.

Proof of Proposition 6.1. Step 1. Proof of (i). It follows from the Lax-Milgram or the Riesz representation theorem that, for all $f \in L_{\rho}^{2}$, there exists a unique solution $u \in H_{\rho}^{1}$ of $\mathcal{L} u+u=f$. Indeed, this equation is equivalent to

$$
(u, w)_{H_{\rho}^{1}} \equiv(\nabla u, \nabla w)+(u, w)=(f, w) \quad \text { for all } w \in H_{\rho}^{1} .
$$

Let $T$ be the solution operator $T: L_{\rho}^{2} \rightarrow L_{\rho}^{2}, f \mapsto u$. Taking $w=u$ in (6.3) and using the CauchySchwarz inequality, we obtain $\|T f\|_{H_{\rho}^{1}} \leq\|f\|$, hence $T$ is continuous. Furthermore, it follows from Lemma 6.3 that $T$ is compact. On the other hand, it is easy to see that $T$ is self-adjoint. It then follows from the spectral theorem that there exists a Hilbert basis of $L_{\rho}^{2}$ made of eigenfunctions of $T$ and this immediately provides the desired result for $\mathcal{L}$.

Step 2. Proof of (ii) for $n=1$ and of (iii). Note that if $\lambda$ is an eigenvalue, then $\lambda \geq 0$ (take $w=v$ in (6.1)). Moreover, the eigenfunctions associated with the eigenvalue $\lambda=0$ are exactly the constant functions. Indeed, they must satisfy $\rho v^{\prime}=C$, hence $C=0$ since $\rho^{-1}=e^{y^{2} / 4} \notin L_{\rho}^{2}$. We next claim that:

$$
\begin{aligned}
& \text { if } v \in H_{\rho}^{1}, v \text { nonconstant, is an eigenfunction with eigenvalue } \lambda \text {, } \\
& \text { then } v^{\prime} \in H_{\rho}^{1} \text { and } v^{\prime} \text { is an eigenfunction with eigenvalue } \lambda-\frac{1}{2} \text {. }
\end{aligned}
$$

Differentiating (6.2), we see that $w:=v^{\prime}$ satisfies $-w^{\prime \prime}+\frac{y}{2} w^{\prime}+\frac{1}{2} w=\lambda w$. The claim will be proved if we check that $w^{\prime} \in L_{\rho}^{2}$. To this end, for all $R>0$, we write

$$
\int_{0}^{R} \rho w^{\prime 2}=\left[\rho w^{\prime} w\right]_{0}^{R}-\int_{0}^{R}\left(\rho w^{\prime}\right)^{\prime} w=\left[\rho w^{\prime} w\right]_{0}^{R}+\left(\lambda-\frac{1}{2}\right) \int_{0}^{R} \rho w^{2} \leq C+\rho w^{\prime} w(R) .
$$


But we necessarily have $\liminf _{y \rightarrow \infty} \rho w^{\prime} w(y)<\infty$, since otherwise we would have in particular $\left(w^{2}\right)^{\prime}=$ $2 w^{\prime} w \geq \rho^{-1}$ for $y \geq y_{0}$ large, hence $w^{2} \geq C+\int_{y_{0}}^{y} e^{t^{2} / 4} \geq C y^{-1} e^{y^{2} / 4}$, hence $\rho w^{2} \geq C y^{-1}$, contradicting $w \in L_{\rho}^{2}$. Consequently, we may pass to the limit along a sequence $R=R_{j} \rightarrow \infty$ to conclude that $\int_{0}^{\infty} \rho w^{\prime 2}<\infty$

Now, since constants are eigenfunctions for $\lambda=0$ only and all eigenvalues are nonnegative, claim (6.4) guarantees that any eigenvalue must be a nonnegative half-integer.

To prove the converse, it suffices to notice that if a polynomial $P$ is an eigenfunction with eigenvalue $\lambda$, then the polynomial $Q(y)=\int_{0}^{y} P(t) d t+\left(\lambda+\frac{1}{2}\right)^{-1} P^{\prime}(0)$ is an eigenfunction with eigenvalue $\lambda+\frac{1}{2}$. Indeed we have $\left[Q^{\prime \prime}+\frac{y}{2} Q^{\prime}-\left(\lambda+\frac{1}{2}\right) Q\right]^{\prime}=Q^{\prime \prime \prime}+\frac{y}{2} Q^{\prime \prime}-\lambda Q^{\prime}=P^{\prime \prime}+\frac{y}{2} P^{\prime}-\lambda P=0$, hence $Q^{\prime \prime}+\frac{y}{2} Q^{\prime}-$ $\left(\lambda+\frac{1}{2}\right) Q=c$ for some constant $c$. But $c=Q^{\prime \prime}(0)-\left(\lambda+\frac{1}{2}\right) Q(0)=P^{\prime}(0)-\left(\lambda+\frac{1}{2}\right) Q(0)=0$.

Finally, the last paragraph guarantees that $E_{k}$ contains a polynomial of degree $k$. It remains to show that $\operatorname{dim}\left(E_{k}\right)=1$. First, $E_{k}$ cannot contain a polynomial $P$ of degree $m>k$, since, by (6.4), $P^{(k)}$ would then be a nonconstant eigenfunction with eigenvalue 0 . It can neither contain two linearly independent polynomials of degree $\leq k$. Indeed, otherwise, by linear combination $E_{k}$ would contain a nonzero polynomial $P$ of degree $m \leq k-1$. But then, by $(6.4), P^{(m)}$ would be a constant eigenfunction with eigenvalue $(k-m) / 2>0$ : a contradiction.

Step 3. Proof of (ii) in the general case and of (iv). For each $\beta \in \mathbb{N}^{n}$, it is immediate to check that $Q_{\beta}$ is an eigenfunction associated with the eigenvalue $\lambda=\sum_{i}^{n} \beta_{i} / 2$. Also we may assume that the family $\left\{P_{j}, j \in \mathbb{N}\right\}$ obtained in Step 2 is orthonormal in $L_{\rho}^{2}(\mathbb{R})$. The $Q_{\beta}$ are then orthonormal in $L_{\rho}^{2}\left(\mathbb{R}^{n}\right)$, since

$$
\int\left(\prod_{i=1}^{n} P_{k_{i}}\left(y_{i}\right)\right)\left(\prod_{i=1}^{n} P_{\ell_{i}}\left(y_{i}\right)\right) e^{-\frac{|y|^{2}}{4}} d y=\prod_{i=1}^{n} \int P_{k_{i}}\left(y_{i}\right) P_{\ell_{i}}\left(y_{i}\right) e^{-\frac{y_{i}^{2}}{4}} d y_{i}=\prod_{i=1}^{n} \delta_{k_{i} \ell_{i}} .
$$

Moreover, it is not difficult to prove that the family $\left\{Q_{\beta}\right\}$ is total. Consequently there can be no other eigenvalue and the result follows.

As a consequence of Proposition 6.1, we can now easily prove Proposition 5.2.

Proof of Proposition 5.2. (i) We write $v=\sum_{k=0}^{\infty} v_{k}$, where $v_{k}$ is the projection of $v$ onto $E_{k}$. Then

$$
\|v\|^{2}=\sum_{k=0}^{\infty}\left\|v_{k}\right\|^{2} \quad \text { and } \quad\|\nabla v\|^{2}=\sum_{k=1}^{\infty} \lambda_{k}\left\|v_{k}\right\|^{2}
$$

Since $v_{1}=0$ due to Remark 6.2 and our assumptions, we deduce that

$$
\|\nabla v\|^{2}=\sum_{k=2}^{\infty} \lambda_{k}\left\|v_{k}\right\|^{2} \geq \lambda_{2} \sum_{k=2}^{\infty}\left\|v_{k}\right\|^{2}=\sum_{k=2}^{\infty}\left\|v_{k}\right\|^{2}=\|v\|^{2}-\left\|v_{0}\right\|^{2} .
$$

Property (5.6) then follows by noting that $v_{0}=\left(\int_{\mathbb{R}^{n}} \rho d y\right)^{-1} \int_{\mathbb{R}^{n}} v \rho d y$, hence $\left\|v_{0}\right\|^{2}=\bar{v}^{2}$. On the other hand, the assumption $\left(v, y_{j}\right)=0$ is clearly satisfied when $v$ is radially symmetric.

(ii) Since $E_{k}$ consists of polynomials of degree $\leq k$ by Proposition 6.1(iii), our assumptions guarantee that $v_{0}=v_{1}=v_{2}=v_{3}=0$. Inequality (5.7) then follows from (6.5), similarly as for assertion (i). Now assume that $v$ is radial, hence $\left(v, y_{j}\right)=0$, and satisfies $(v, 1)=\left(v,|y|^{2}\right)=0$. By symmetry, we have $\left(v, y_{j}^{2}\right)=\frac{1}{n}\left(v,|y|^{2}\right)=0$ for all $j$. Moreover, using radial symmetry again, we easily obtain $\left(v, y_{j} y_{k}\right)=0$ for all $j \neq k$ and $\left(v, y_{j} y_{k} y_{\ell}\right)=0$ for all $j, k, \ell$. It follows that (5.7) is true.

(iii) Integrating by parts and using our assumptions, we obtain $\int_{\mathbb{R}^{n}} \rho \partial_{y_{i}} v d y=-\int_{\mathbb{R}^{n}} v \partial_{y_{i}} \rho d y=$ $\frac{1}{2} \int_{\mathbb{R}^{n}} v y_{i} \rho d y=0$ and, for all $j \in\{1, \ldots, n\}$,

$$
\int_{\mathbb{R}^{n}} y_{j} \rho \partial_{y_{i}} v d y=-\int_{\mathbb{R}^{n}} v \partial_{y_{i}}\left(y_{j} \rho\right) d y=\int_{\mathbb{R}^{n}} v\left(\frac{1}{2} y_{i} y_{j}-\delta_{i j}\right) \rho d y=0
$$


(integration by parts can be justified by a similar argument as in the proof of Proposition 5.1). Inequality (5.8) thus follows by applying (5.6) to $\partial_{y_{i}} v$. The last statement follows by noting that, for all $i$, such radial $v$ satisfies $\left(v, y_{i}\right)=0,\left(v, y_{i}^{2}-2\right)=\frac{1}{n}\left(v,|y|^{2}-2 n\right)=0$ and $\left(v, y_{i} y_{j}\right)=0$ for all $j \neq i$.

\section{References}

[1] J. Bebernes and S. Bricher, Final time blowup profiles for semilinear parabolic equations via center manifold theory, SIAM J. Math. Anal. 23 (1992), 852-869.

[2] J. Bricmont and A. Kupiainen, Universality in blow-up for nonlinear heat equations, Nonlinearity 7 (1994), 539-575.

[3] Y.-G. Chen, Blow-up solutions of a semilinear parabolic equation with the Neumann and Robin boundary conditions, J. Fac. Sci. Univ. Tokyo Sect. IA Math. 37 (1990), 537-574.

[4] X. Chen, M. Fila and J.-S. Guo, Boundedness of global solutions of a supercritical parabolic equation, Nonlinear Anal. 68 (2008), 621-628.

[5] S. Filippas and R. Kohn, Refined asymptotics for the blowup of $u_{t}-\Delta u=u^{p}$, Comm. Pure Appl. Math. 45 (1992), 821-869.

[6] S. Filippas and W.-X. Liu, On the blowup of multidimensional semilinear heat equations, Ann. Inst. H. Poincaré Anal. Non Linéaire 10 (1993), 313-344.

[7] A. Friedman and B. McLeod, Blow-up of positive solutions of semilinear heat equations, Indiana Univ. Math. J. 34 (1985), 425-447.

[8] V.A. Galaktionov and S. Posashkov, The equation $u_{t}=u_{x x}+u^{\beta}$. Localization, asymptotic, Akad. Nauk SSSR Inst. Prikl. Mat. Preprint no. 97 (1985).

[9] V.A. Galaktionov and S. Posashkov, Application of new comparison theorems to the investigation of unbounded solutions of nonlinear parabolic equations (Russian), Differentsial'nye Uravneniya 22 (1986), 1165-1173, (English translation: Differential Equations 22 (1986), 809-815).

[10] Y. Giga and R.V. Kohn, Asymptotically self-similar blow-up of semilinear heat equations, Comm. Pure Appl. Math. 38 (1985), 297-319.

[11] Y. Giga and R.V. Kohn, Characterizing blowup using similarity variables, Indiana Univ. Math. J. 36 (1987), 1-40.

[12] Y. Giga and R.V. Kohn, Nondegeneracy of blowup for semilinear heat equations, Comm. Pure Appl. Math. 42 (1989), 845-884.

[13] M.A. Herrero and J.J.L. Velázquez, Blow-up profiles in one-dimensional, semilinear parabolic problems, Comm. Partial Differential Equations 17 (1992), 205-219.

[14] M.A. Herrero and J.J.L. Velázquez, Blow-up behaviour of one-dimensional semilinear parabolic equations, Ann. Inst. H. Poincaré Anal. Non Linéaire 10 (1993), 131-189.

[15] M.A. Herrero and J.J.L. Velázquez, A blow up result for semilinear heat equations in the supercritical case (1994), Preprint.

[16] L.A. Lepin, Countable spectrum of eigenfunctions of a nonlinear heat-conduction equation with distributed parameters, Differentsial'nye Uravneniya 24 (1988), 1226-1234, (English translation: Differential Equations 24 (1988), 799-805).

[17] L.A. Lepin, Self-similar solutions of a semilinear heat equation, Mat. Model. 2 (1990), 63-74, (in Russian).

[18] W.-X. Liu, Blow-up behavior for semilinear heat equations: multi-dimensional case, Rocky Mountain J. Math. 23 (1993), 1287-1319.

[19] H. Matano and F. Merle, On nonexistence of type II blowup for a supercritical nonlinear heat equation, Comm. Pure Appl. Math. 57 (2004), 1494-1541. 
[20] H. Matano and F. Merle, Classification of type I and type II behaviors for a supercritical nonlinear heat equation, J. Funct. Anal. 256 (2009), 992-1064.

[21] H. Matano and F. Merle, Threshold and generic type I behaviors for a supercritical nonlinear heat equation, J. Funct. Anal. 261 (2011), 716-748.

[22] J. Matos, Self-similar blow up patterns in supercritical semilinear heat equations, Comm. Appl. Anal. 5 (2001), 455-483.

[23] F. Merle and H. Zaag, Optimal estimates for blowup rate and behavior for nonlinear heat equations, Comm. Pure Appl. Math. 51 (1998), 139-196.

[24] F. Merle and H. Zaag, Refined uniform estimates at blow-up and applications for nonlinear heat equations, Geom. Funct. Anal. 8 (1998), 1043-1085.

[25] N. Mizoguchi, Type-II blowup for a semilinear heat equation, Adv. Differential Equations 9 (2004), 1279-1316.

[26] N. Mizoguchi, Nonexistence of backward self-similar blowup solutions to a supercritical semilinear heat equation, J. Funct. Anal. 257 (2009), 2911-2937.

[27] N. Mizoguchi, On backward self-similar blow-up solutions to a supercritical semilinear heat equation, Proc. Roy. Soc. Edinburgh Sect. A 140 (2010), 821-831.

[28] N. Mizoguchi, Non-existence of type II blowup solutions for a semilinear heat equation, J. Differential Equations 250 (2011), 26-32.

[29] P. Poláčik, P. Quittner and Ph. Souplet, Singularity and decay estimates in superlinear problems via Liouville-type theorems. Part II: parabolic equations, Indiana Univ. Math. J. 56 (2007), 879908.

[30] P. Quittner and Ph. Souplet, Superlinear Parabolic Problems. Blow-up, Global Existence and Steady States, Birkhäuser Advanced Text, Basel, 2007.

[31] J.J.L. Velázquez, Higher dimensional blow up for semilinear parabolic equations, Comm. Partial Differential Equations 17 (1992), 1567-1596.

[32] J.J.L. Velázquez, Classification of singularities for blowing up solutions in higher dimensions, Trans. Amer. Math. Soc. 338 (1993), 441-464.

[33] J.J.L. Velázquez, Estimates on the $(n-1)$-dimensional Hausdorff measure of the blow-up set for a semilinear heat equation, Indiana Univ. Math. J. 42 (1993), 445-476.

[34] F.B. Weissler, Single point blow-up for a semilinear initial value problem, J. Differential Equations 55 (1984), 204-224.

[35] F.B. Weissler, An $L^{\infty}$ blow-up estimate for a nonlinear heat equation, Comm. Pure Appl. Math. 38 (1985), 291-295.

[36] H. Zaag, Determination of the curvature of the blow-up set and refined singular behavior for a semilinear heat equation, Duke Math. J. 133 (2006), 499-525. 